\title{
Characterisation of the potential function of SVA retrotransposons to modulate gene expression patterns
}

\author{
Abigail L Savage1, Vivien J Bubb¹, Gerome Breen ${ }^{2,3}$ and John P Quinn ${ }^{1 *}$
}

\begin{abstract}
Background: Retrotransposons are a major component of the human genome constituting as much as $45 \%$. The hominid specific SINE-VNTR-Alus are the youngest of these elements constituting $0.13 \%$ of the genome; they are therefore a practical and amenable group for analysis of both their global integration, polymorphic variation and their potential contribution to modulation of genome regulation.

Results: Consistent with insertion into active chromatin we have determined that SVAs are more prevalent in genic regions compared to gene deserts. The consequence of which, is that their integration has greater potential to have affects on gene regulation. The sequences of SVAs show potential for the formation of secondary structure including G-quadruplex DNA. We have shown that the human specific SVA subtypes (E-F1) show the greatest potential for forming G-quadruplexes within the central tandem repeat component in addition to the $5^{\prime}$ ' $C C C T C T$ ' hexamer. We undertook a detailed analysis of the PARK7 SVA D, located in the promoter of the PARK7 gene (also termed $D J-1$ ), in a HapMap cohort where we identified 2 variable number tandem repeat domains and 1 tandem repeat within this SVA with the $5^{\prime}$ CCCTCT element being one of the variable regions. Functionally we were able to demonstrate that this SVA contains multiple regulatory elements that support reporter gene expression in vitro and further show these elements exhibit orientation dependency.
\end{abstract}

Conclusions: Our data supports the hypothesis that SVAs integrate preferentially in to open chromatin where they could modify the existing transcriptional regulatory domains or alter expression patterns by a variety of mechanisms.

Keywords: SVA, PARK7, DJ-1, Genetic variation, G-quadruplex DNA, Retrotransposon

\section{Background}

Mobile DNA, such as long terminal repeats (LTRs), long interspersed elements (LINEs), short interspersed elements (SINEs) and SINE-VNTR-Alus (SVAs), constitute up to $45 \%$ of the human genome. These retrotransposable elements are mobilised via a 'copy and paste' mechanism; namely a RNA intermediate is reverse transcribed into DNA which inserts back into the genome at a different loci to the source sequence. Historically SVAs were originally identified as a sequence derived from part of the env gene and a 3'LTR from the HERV-K10 endogenous retrovirus with a poly A-tail and a GC-rich tandem repeat directly upstream and were named SINE-R elements [1]. It

\footnotetext{
* Correspondence: jquinn@liv.ac.uk

${ }^{1}$ Department of Molecular and Clinical Pharmacology, Institute of

Translational Medicine, The University of Liverpool, Liverpool L69 3BX, UK

Full list of author information is available at the end of the article
}

was later shown that in the $\mathrm{C} 2$ gene, the GC tandem repeat of the SINE-R element was a variable number tandem repeat (VNTR) [2]. This composite element was termed a SINE-VNTR-Alu (SVA) when further analysis of its components revealed the Alu-like sequences adjacent to the VNTR [3]. Thus typically SVAs consist of a hexamer repeat (CCCTCT), an Alu-like sequence, a GC-rich VNTR, a SINE and a poly A-tail.

Such SVAs, which are hominid specific, are to date the smallest of the retrotransposon families identified with 2676 elements found in the Hg19 amounting to $0.13 \%$ of the genome. A precursor of the VNTR domain found within the SVAs is present within the rhesus macaque genome, many of these precursor elements are also present in the human genome suggesting they were retrotransposing prior to the divergence of the old world monkeys and the hominoids [4]. SVAs are divided into

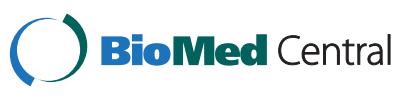

(c) 2013 Savage et al.; licensee BioMed Central Ltd. This is an Open Access article distributed under the terms of the Creative Commons Attribution License (http://creativecommons.org/licenses/by/2.0), which permits unrestricted use, distribution, and reproduction in any medium, provided the original work is properly cited. 
subtypes (A-F) by the SINE region and their age estimated at $13.56 \mathrm{Myrs}$ old for the oldest subtype (A) and 3.18Myrs old for the youngest subtype (F) [5]. A seventh subtype has been identified that contains a 5 ' transduction of the sequence from the first exon of the MAST2 gene and associated $\mathrm{CpG}$ island and has been referred to as either CpG-SVA, MAST2 SVA or SVA F1 [6-8]. The sequence of the MAST2 loci that has been incorporated into the F1 structure has been shown to act as a positive regulator of transcription in a reporter gene construct when transfected into human germ cells and is thought to have contributed to the success of the subtype in its retrotransposition [9]. Subtypes E, F and F1 are human specific as are some members of SVA subtype D. The younger subtypes appear to contain two GC rich VNTRs as opposed to the one seen in the older subtypes.

SVAs are non autonomous and are mobilised by the LINE-1 protein machinery $[10,11]$, their retrotransposition rate is estimated at 1 in every 916 births [12]. A recent study to determine the nature of SVA retrotransposition revealed that no individual domain of an SVA is fundamental for this to occur, but each domain differentially affected the rate at which retrotransposition can take place [13]. To date eight SVA insertions have been associated with disease $[14,15]$, these include for example a SVA in the 3'UTR of the fukutin gene which causes Fukyama-type congenital muscular dystrophy by decreasing mRNA production, and a SVA insertion and subsequent $14 \mathrm{~kb}$ deletion of the HLA-A gene locus linked with leukaemia $[16,17]$. Retrotransposition events are repressed in somatic cells via epigenetic modifications and post transcriptional suppression but there is recent evidence for these events occurring in the adult brain and their insertions are associated with protein coding genes active in the brain [18]. In tumour cells, SVAs along with other retrotransposons become demethylated and potentially could lose the epigenetic modifications that silenced them [19]. The latter indicates that retrotransposons including SVAs could modify the genomic structure of a locus with associated consequences for regulation without the requirement for retrotransposition.

The nature of the sequence contained within SVAs shows the potential for formation of secondary structures such as cruciforms and G-quadruplexes (G4) [20]. G4 DNA is a secondary structure predicted from bioinformatic analysis to form in guanine-rich sequences, but validation in vivo is difficult and highly debated [21-23]. G4 structures are hypothesised to interfere with replication of DNA and be involved in a host of regulatory functions including gene expression, genome stability and telomerase activity [24-27].

SVAs contain large domains of repetitive DNA (VNTRs) similar in copy number and size of individual repeats to those, we and others, have found to direct differential tissue specific and stimulus inducible gene expression in many other genes [28-35]. This differential regulator property has been correlated with copy number of the VNTR in some genes [30,34-43]. For example, we and others have demonstrated that VNTRs located in the promoter and second intron of the human serotonin transporter gene (SLC6A4) are differential as both risk factors for mental health and tissue specific regulators in the context of reporter gene constructs, in vivo and in vitro based on the copy number of the repeat $[29,31,35]$.

In this communication we have determined the global location of SVAs, and then focused on the individual variation and function of a single selected SVA located in the PARK7 gene promoter. The PARK7 SVA was chosen because it is human specific, was regarded as a complete SVA and because of the nature of its location in relation to the PARK7 gene. We addressed the potential function of this SVA as a transcriptional regulator, by investigating its activity in a reporter gene construct.

\section{Results}

Distribution of SVA elements across the human genome

The SVA density of each chromosome was found to be positively correlated with gene density $(r=0.74)$ as shown in Figure 1A (for values for each chromosome see Additional file 1). The correlation coefficient for the relationship between gene density and SVA density was calculated using the bootstrap confidence interval (95\%) to remove outliers. However when the density of each SVA subtype was analysed individually, a negative correlation with gene density across chromosomes was found for subtype A, whereas all other subtypes showed a positive correlation (see Additional file 2). The oldest of the subtypes, A, show a clear difference in the pattern of insertion in the genome to the rest of this family of retrotransposons, however the mechanism behind this is unclear.

To dissect the distribution of SVAs further, the genome was divided into the three following regions: genes, intergenic and gene deserts and the observed distribution of members of the four classes of retrotransposon (LTRs, LINEs, SINEs and SVAs) was compared to the expected. The expected number of each element was determined by calculating the number of elements that would be present in the region in relation to its size if the elements inserted randomly throughout the genome. Gene deserts were defined as regions between genes which were $250 \mathrm{~kb}$ away from the start or end of a known gene, intergenic as regions between genes that are less than $250 \mathrm{~kb}$ from the start or end of a known gene and genes were determined by the UCSC gene track from the UCSC genome browser. The regions were defined in this manner to assess if the retrotransposons had preferentially 


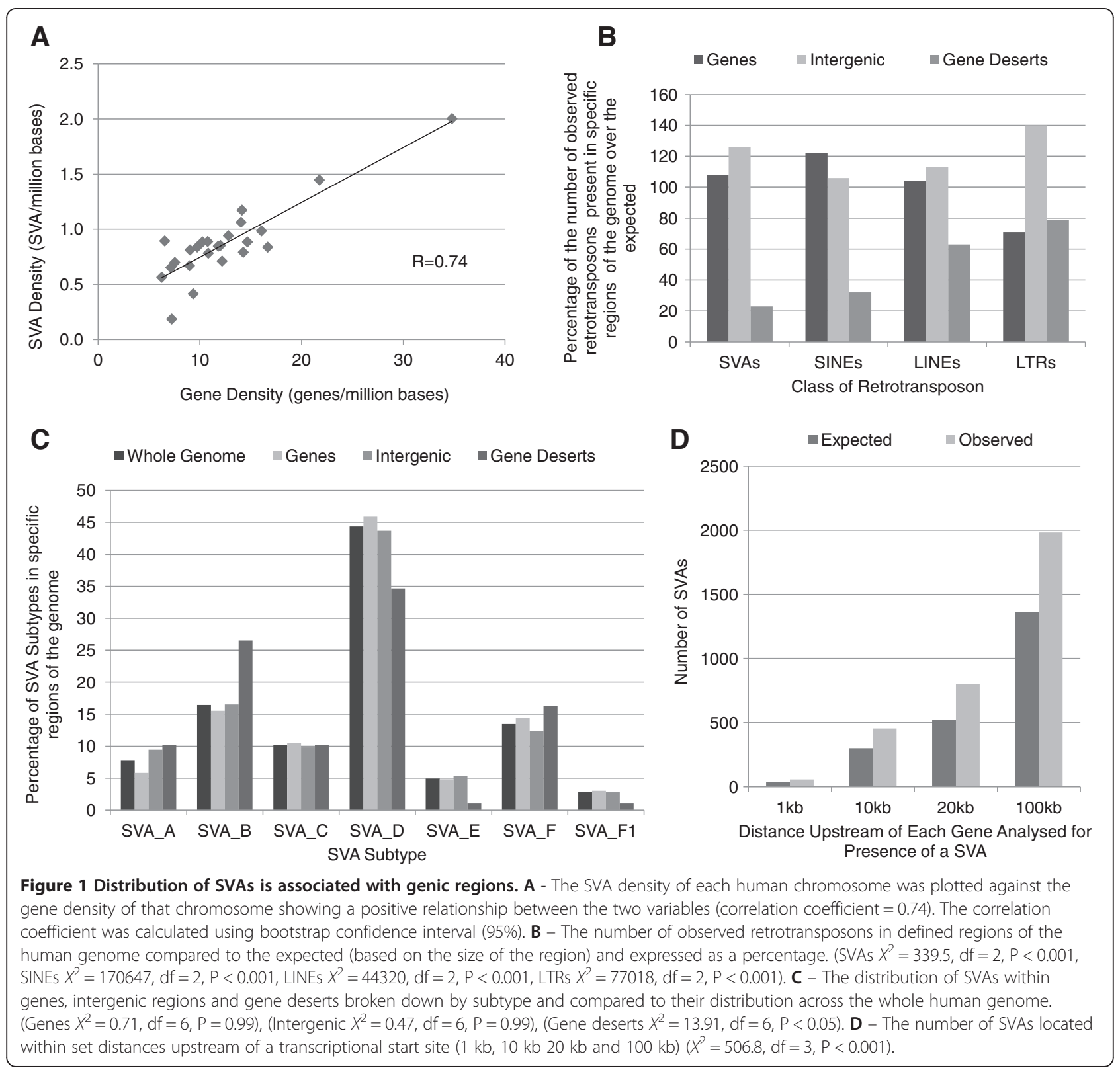

inserted into regions devoid of genes (gene deserts) or regions of the genome that could include active chromatin where genes and intergenic regions potentially containing regulatory domains (up to $250 \mathrm{~kb}$ from TSS) are located $[44,45]$. The distribution of the different classes of retrotransposons shared some similarities, in particular a lower number than expected were found in gene deserts and all classes showed a significant difference in their actual distribution to the expected across the three regions analysed, Figure $1 \mathrm{~B}$ (SVAs $X^{2}=339.5, \mathrm{df}=2, \mathrm{P}<0.001$, SINEs $X^{2}=170647, \mathrm{df}=2, \mathrm{P}<0.001$, LINEs $X^{2}=44320$, $\mathrm{df}=2, \mathrm{P}<0.001$, LTRs $\left.X^{2}=77018, \mathrm{df}=2, \mathrm{P}<0.001\right)$. The distribution of SVAs was further analysed by subtype within the previously defined regions: genes, intergenic and gene deserts (Figure 1C). The SVA subtypes showed a significant difference in their distribution within gene deserts compared to the whole genome (Gene deserts $X^{2}=$ 13.91, $\mathrm{df}=6, \mathrm{P}<0.05)$ but not within genes and intergenic regions (Genes $X^{2}=0.71, \mathrm{df}=6, \mathrm{P}=0.99$, Intergenic $X^{2}=$ $0.47, \mathrm{df}=6, \mathrm{P}=0.99)$. Subtypes $\mathrm{D}, \mathrm{E}$ and $\mathrm{F} 1$ were underrepresented in gene deserts whereas subtype B in particular was found in higher numbers. The SVAs also showed a significant increase in regions 1-100 kb directly upstream of transcriptional start sites when the observed number was compared to the expected for the size of these regions $\left(X^{2}=506.8, \mathrm{df}=3, \mathrm{P}<0.001\right)$ (Figure $\left.1 \mathrm{D}\right)$. The subtype distribution was significantly different within the first kilobase upstream of the start of transcription 
(see Additional file 3); subtypes A, B and E were found in lower numbers than expected and there were a greater number of subtypes $C$ and $D$.

\section{Potential of SVA subtypes to form G-quadruplexes}

We investigated the potential of SVAs, more specifically the CCCTCT hexamer repeat at the 5' end and the more central VNTR region, to form G4 DNA. Of the total genomic DNA that can form G4 DNA (predicted by Quadparser software [46]) $1.88 \%$ is due to SVAs which only constitute $0.13 \%$ of the human genome. When repetitive or mobile DNA elements, which include simple repeats, microsatellites, LTRs, LINEs, SINEs and DNA transposons (as defined by UCSC genome browser Hg19 http://genome.ucsc.edu/index.html) are compared; SVAs have the greatest potential contribution to G4 DNA for their size for any specific element (Figure 2A). The sequence of the PARK7 SVA is shown in Figure 3 with the bases that contribute to its G4 potential predicted by Quadparser software in italics.

It was found that the percentage of sequence in each SVA subtype with the potential to form G4 increased as the age of the subtype decreased, thus subtypes E, F and F1 have the greatest potential for G4 formation (Figure 2B). This can be explained by the increase in the potential of the central VNTR region to form G4 DNA from subtype D through to F1. The possible amount of G4 formed by the CCCTCT repeat was found to increase through subtypes A

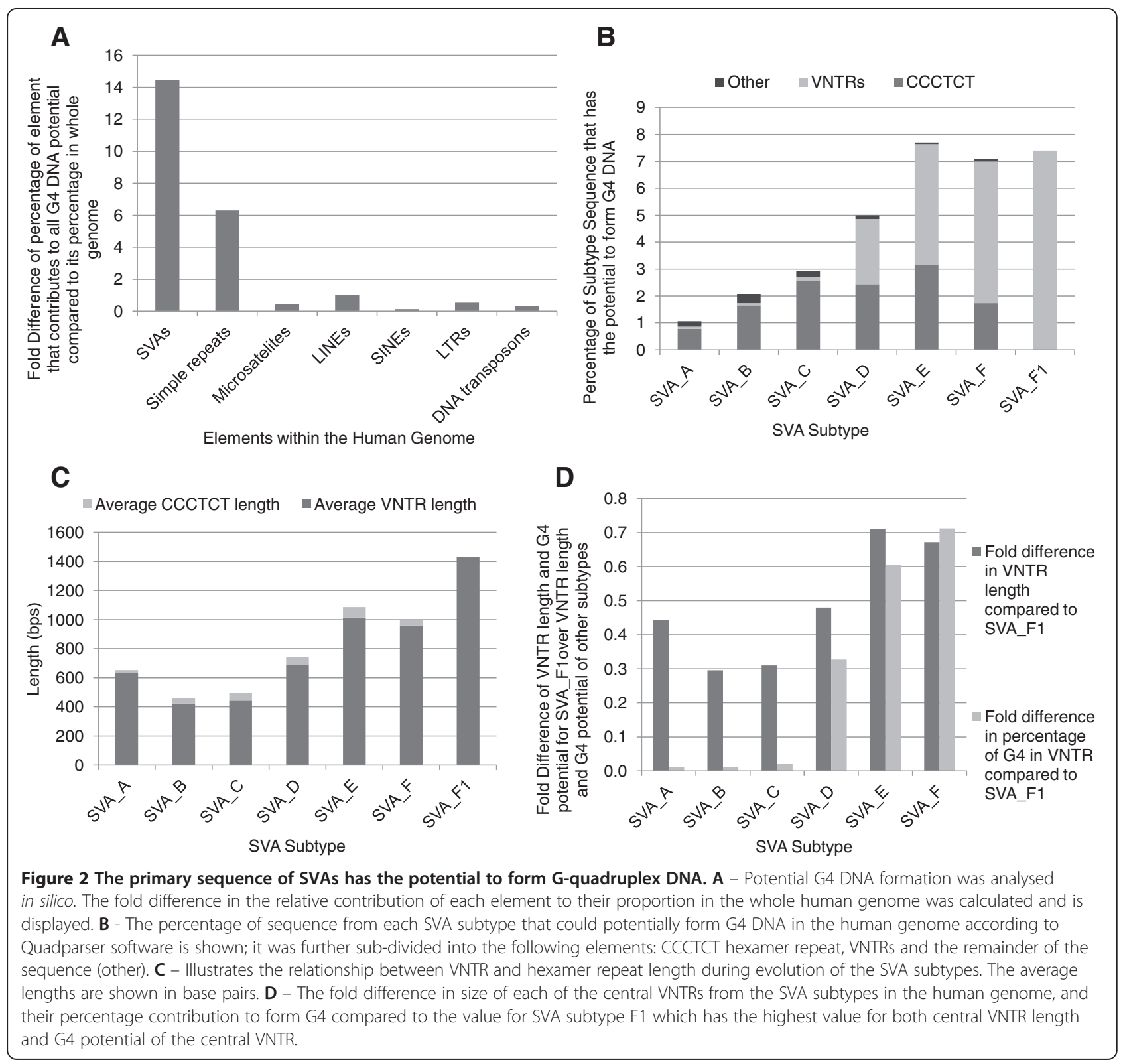




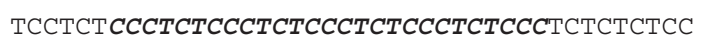

\section{Hexamer VNTR}

ACGGTCTCCTTCCACGGTCTCCCTCTGATGCCGAGCCAAAGCTGGACGGTACTGCTGCC ATCTCGGCTCACTGCAACCTCCCTGCCTGATTCTCCTGCCTCAGCCTGCCGAGTGCCTG CGCA $\overline{C G C C G C C A C G C C T G A C T G G T T T T C G T T T T T T T T T T T T G T G G A G A C G G G G T T T T G C ~}$ TGTGTTGGCCGGGCTGGTCTCCAGCTCCTAACCACGAGTGATCCGCCAGCCTCGGCCTC CCGAGGTGCCGGGATTGCAGACGGAGTCTCGTTCACTCAGTGCTCAATGGTGCCCAGGC TGGAGTGCA GTGGCGTGATCT $\overline{C G}$ GTCGCTACAACCTCCACCTCCCAGCCGCCTGCCTT GGCCCCCCAA
\end{abstract}

\begin{abstract}
AGTGCCGAGATTGCAGCCTCTGCCCAGCCGCCACCCCGTC

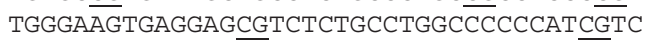
TGGGATACGAGGAGCCTCTCTGCCTGGCTGCCCAGTC TGGAAAGTGAGGAGCGTCCCTGCCCGGCCGCCATCCCATC

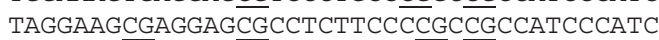
TAGGAAGTGAGGAGCGTCTCTGCCCGGCCACCCATCGTC TGAGATGTGGGGAG $\overline{C A} C C T C T G C C \overline{C C}$ GCGCCCTGTC TGGGATGTGAGGAGCGCCTCTGCTGGGCCGCAACCCTGTC TGGGAGGTGAGGAG $\overline{C G T C T C T G C C C G G C \overline{C G} C C C C G T C}$ TGAGAAGTGAGAAAACCCTCTGCCTGGCAACCG CCCCGTC TGAGAAGTGAGGAGCCCCTCCGTCCGGCAGCCACCC CGTC

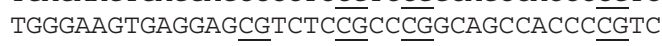

TGGGAGGGAGGTGGGGGGGGGGTCAGCCCCCTGCCCGGCCAGCTGCCCTGTC CGGGAGGTGAGGGGCTCCTCTGCCCGGCCAGCCGCCCCGTC

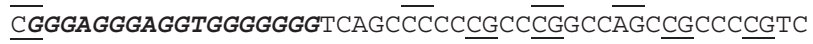

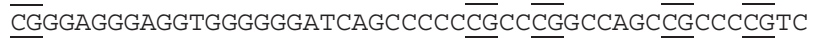
CG GGAGGGAGGTGGGGGGGTCAGCCCCC $\overline{C C}$ CCCGGCCAGCCGCCCTATC

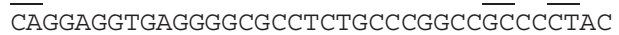
TGGGAAGTGAGGAGCCCCTCTGCCTGGCDAGCCGCCCCGTC CGGGAGGGTGGTGGGGGGGTCAGCCCCCCGCCCGGCCAGCCGCCCCATC CGGGAGGTGAGGGGCGCTTCTGCCCGGCDGCC $\overline{C C T A C}$

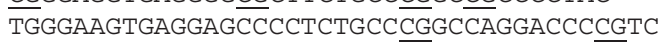

TGGGAGGTGTGCCCAGCGGCTCATTGGGGATGGGCCATGATGACAATGGCGGTTTTGTG GAATAGAAAGGCGGGAA $\overline{A G G T G G G G A A A A A A T T G A G A A A T C G G A T G G T T G C C G G G T C T G ~}$ TGTGGATAGAAGTAGACATGGGAGACTTTTCATTTTGTTTTGTACTAAGAAAAATTTTT TTGCCTTGGAAAAAAAAAAAAAAAAAAAAAAA

\section{Alu-Like \\ sequence}

VNTR

\section{SINE and poly A tail}

Figure 3 Primary sequence of allele 1 of PARK7 SVA identifying the different components. The human-specific PARK7 SVA located $8 \mathrm{~kb}$ upstream of the PARK7 gene (chr1:8012112-8013618) contains a CCCTCT hexamer VNTR, Alu-like sequence, TR, VNTR, SINE and poly A-tail. In italics are the sequences of DNA that have been predicted to have the potential to form G4 DNA by Quadparser software, potential sites of methylation (CpGs) are underlined.

to E; however the proportion it contributed to the total G4 potential of each subtype decreased. Subtype F1 does not contain a CCCTCT repeat therefore all of its G4 potential is within the central VNTR.

The average number of repeats in the CCCTCT domain varied between subtypes (Figure $2 \mathrm{C}$ ) which accounts for the difference in G4 potential between the SVA subtypes in this particular domain; the longer the CCCTCT domain the greater the G4 potential. The average length of the GC rich VNTRs also varied between subtypes but length did not show the same direct correlation with G4 potential as in the CCCTCT domain. For example the VNTRs of subtype A are just under half the length of those of subtype F1, however they have only a hundredth of the potential to form G4 DNA when compared to the VNTR sequences of subtype F1 (Figure 2D). It appears that the subtypes fall into two main groups when analysing the G4 potential in the VNTRs. Subtypes A, B and C have very low
G4 potential in their VNTRs compared to subtypes E, F and F1 with subtype D bridging the difference between the older hominid specific and younger human specific subtypes. This can be explained by the development of the additional second VNTR of the younger subtypes with differences in the primary nucleotide content to the first VNTR containing sequences that have the potential for G4 DNA (Figure 3).

\section{Genetic variation of PARK7 SVA}

We analysed in detail the primary sequence and repeat variation in the human specific SVA D found upstream of the PARK7 gene. The PARK7 SVA is located $8 \mathrm{~kb}$ upstream of the $P A R K 7$ major transcriptional start site defined by both the UCSC browser (http://genome. ucsc.edu/index.html Hg19) and the literature [47]. A putative alternative $P A R K 7$ transcript also exists, that would originate within $1 \mathrm{~kb}$ of this SVA based on 
expressed sequence tags and other data in the UCSC browser and Archive ensembl (ensembl10:Jan2013). Genotypic analysis of this SVA identified four distinct alleles which were polymorphic in length, in 87 individuals from the CEU (Utah residents with Northern and Western European ancestry from the CEPH collection) HapMap cohort with allelic frequencies shown in Table 1. Alleles 1 and 3 were the most common within this cohort with $94 \%$ of the individuals having at least one of these alleles. The primary sequence of allele 1 of the PARK7 SVA is shown in Figure 3 with the different domains, VNTRs, SINE and Alu-like, identified. Figure 3 also shows the CpGs underlined and the bases that contribute to the PARK7 SVA's G4 potential in italics. Allelic variation was found to be generated by differences in the number of repeat units present of specific repetitive elements within the SVA, namely the CCCTCT hexamer repeat and in the most 3 ' of the two large central VNTRs. VNTR variation within the cohort was analysed by PCR and confirmed by a more limited sequence analysis of specific variants. The hexamer domain was either a 7, 10 or 13 repeat domain, and the second VNTR consisted of either 10,11 or 12 repeats with a repeat length of 37-52 bp in this cohort. We observed no variation in the number of repeats in the most 5 ' of the central 'VNTRs', which was a stable 12 copy variant of 37-40 bp repeat length, which was therefore termed a tandem repeat (TR). Schematic in Figure 4A shows the structure of the complete PARK7 SVA and the variation found in its repetitive regions is summarised in Table 2.

Functional activity of PARK7 SVA in reporter gene analysis We addressed whether both the intact PARK7 SVA and its distinct individual domains could act as transcriptional regulators. SVAs can be found in the same, or opposite

Table 1 Frequency of each allelotype for the PARK7 SVA in the HapMap cohort

\begin{tabular}{ccc}
\hline Genotype & Number of samples & $\begin{array}{c}\text { Percentage of allelotype } \\
\text { within Hap Map samples }\end{array}$ \\
\hline $1 / 1$ & 19 & 21.8 \\
$1 / 2$ & 4 & 4.6 \\
$1 / 3$ & 35 & 40.2 \\
$1 / 4$ & 4 & 4.6 \\
$2 / 2$ & 4 & 4.6 \\
$2 / 3$ & 3 & 3.4 \\
$2 / 4$ & 1 & 1.1 \\
$3 / 3$ & 16 & 18.4 \\
$3 / 4$ & 1 & 1.1 \\
$4 / 4$ & 0 & 0.0 \\
Total & 87 & \\
\hline
\end{tabular}

The alleles are numbered 1-4 from shortest to longest. orientation to the gene they are located near to. When analysed, $49 \%$ of the SVAs found within $10 \mathrm{~kb}$ upstream of transcriptional start sites were on the same strand as the gene, for these reasons we also tested whether their function was orientation dependant. Eight reporter gene constructs were generated (Figure 4A) containing the following fragments in both forward and reverse orientations:

- the whole SVA (SVA)

- SVA with the SINE region deleted (SVA wo SINE)

- central TR and VNTR (TR/VNTR)

- a 5 ' truncation with only the CCCTCT hexamer, Alulike sequence and 10 of the 12 repeats of the TR of allele 1 of the PARK7 SVA (truncated SVA) present

SVAs are described as having a CCCTCT domain at their 5' end and a poly A-tail at their 3' end therefore this was used to define the forward orientation. We compared the ability of the eight fragments to support reporter gene expression (luciferase) directed by a heterologous minimal promoter in two cell lines SK-N-AS, a human neuroblastoma cell line and MCF-7, a human breast cancer cell line.

In the SK-N-AS cell line (Figure 4B) the intact PARK7 SVA in forward orientation did not alter the levels of reporter gene expression, when compared to the minimal promoter alone (pGL3P) however when the SINE domain was deleted reporter gene activity was significantly enhanced $(p<0.05)$. The TR/VNTR and the truncated SVA in the forward orientation acted to significantly repress luciferase activity when compared to the minimal promoter alone ( $\mathrm{pGL3P})(\mathrm{p}<0.001, \mathrm{p}<0.01$ respectively). When the domains were tested in the reverse orientation the reporter gene levels were all significantly different when compared to the levels seen in the forward orientation (SVA $\mathrm{p}<0.001$, SVA wo SINE $\mathrm{p}<0.05$, TR/VNTR $\mathrm{p}<0.05$, truncated SVA $\mathrm{p}<0.01$ ). The activity of the SVA and SVA wo SINE in reverse orientation were reduced compared to when in the forward orientation whereas the activity of the TR/VNTR and truncated SVA showed the opposite trend.

The reporter gene constructs showed distinct activity levels in the MCF-7 cell line when compared to that observed in the SK-N-AS cell line (Figure 4C). In forward orientation the complete SVA had a significant increase in reporter activity in MCF-7 cells $(\mathrm{p}<0.01)$, distinct from its function in SK-N-AS, however similarly to SKN-AS cells the SVA wo SINE showed the greatest ability to enhance reporter gene activity. In contrast the TR/ VNTR showed similar activity to that of the minimal promoter alone. The truncated SVA acted as a repressor as it did in the SK-N-AS cell line $(\mathrm{p}<0.05)$. The domains in the reverse orientation all showed a significant difference to the activity of the domains in the forward 


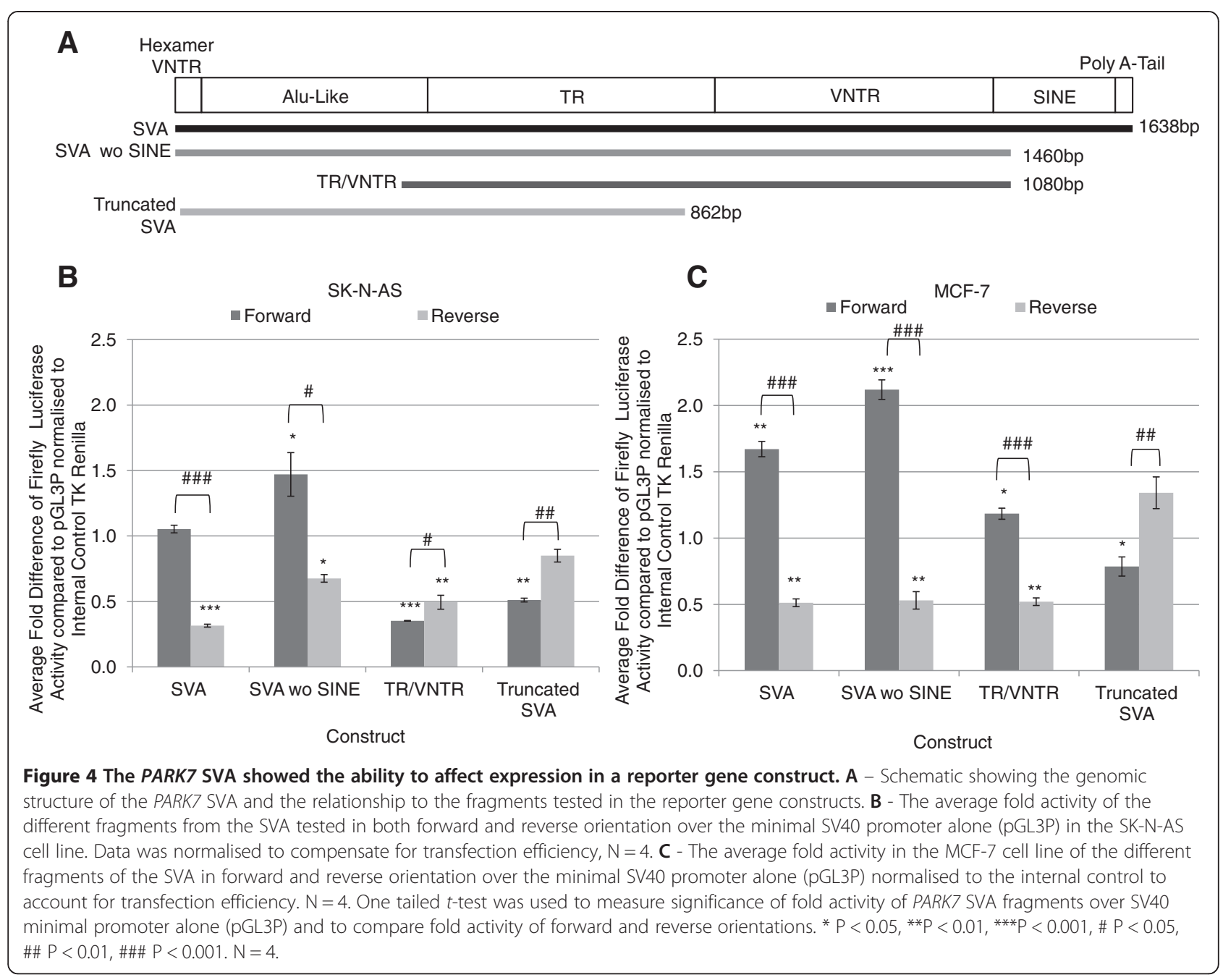

orientation (SVA $\mathrm{p}<0.001$, SVA wo SINE $\mathrm{p}<0.001$, TR/ VNTR $\mathrm{p}<0.001$, truncated SVA $\mathrm{p}<0.01)$. The SVA, SVA wo SINE and TR/VNTR all showed decreased activity in the reverse orientation when compared to the domains in the forward orientation. The truncated SVA showed

Table 2 Sequence analysis of the four alleles identified in the PARK7 SVA

\begin{tabular}{cccc}
\hline & \multicolumn{3}{c}{ Number of repeats } \\
\hline Alleles of PARK7 SVA & Hexamer VNTR & TR & VNTR \\
1 & 7 & 12 & 10 \\
2 & 10 & 12 & 11 \\
3 & 10 & 12 & 12 \\
4 & 13 & 12 & 12 \\
\hline
\end{tabular}

Genomic DNA from individuals in the CEU (Utah residents with Northern and Western European ancestry from the CEPH collection) HapMap cohort was analysed. The length variation detected occurred in the CCCTCT hexamer repeat (termed hexamer VNTR) and within a second repetitive VNTR region further downstream. In this cohort a repetitive domain here termed a TR was not found to vary between the individuals within this population; this TR was located upstream of the second VNTR. The alleles were numbered 1-4 from shortest to longest. One example of each type of allele was sequenced. greater activity in the reverse orientation than when in the forward orientation.

\section{Discussion}

Retrotransposons, including SVAs, can affect gene function by multiple mechanisms particularly when inserted into protein coding regions $[48,49]$. They have also been suggested to modulate transcriptional and posttranscriptional parameters based partially on their location within introns and promoters, however the functional significance of these non coding integrations is much more difficult to determine than those in exons. Epigenetic silencing which suppresses retrotransposition in somatic cells might have modulator effects on transcriptional or post transcriptional domains adjacent to sites of integration. Removal of such epigenetic silencing may correlate with retrotransposition in the aging CNS [18] and the observed hypomethylated state of SVAs in cancer [19]. This may suggest the potential for a dynamic chromatin structure over the locus of the SVAs under specific environmental conditions and challenges. In either 
circumstance the SVAs have the potential to influence the local genome architecture via epigenetic modifications, the formation of secondary structures and the binding of sequence specific transcription factors to the SVA.

Using the most recent version of the human genome, Hg19, we have demonstrated a minimum of 2676 SVA insertions in the human genome. This is considerably less in number than seen in the other classes of retrotransposons; this can be explained by the fact that they are the most recent family to integrate and proliferate in the genome. It is also likely that the primary DNA sequence of the members of this family has undergone the least number of alterations which may also suggest SVAs share related biochemical and functional properties. These properties will in part be directed by the primary sequence of the SVA to allow for such as interaction with transcription factors and other modulators of genome function acting as sequence specific binding proteins. A further regulatory function of the SVA could be directed by the genomic structure adopted upon insertion. Superimposed on these regulatory parameters could be modulation of their activity by the polymorphic nature of the distinct domains within the SVA such as the VNTR elements. There is an extensive literature on VNTR domains both being differentially associated with disease and transcriptional properties based on the copy number of the repeats $[29,50]$. In this study we addressed firstly the site of integration of SVA elements, secondly the potential secondary structures formed and finally a detailed analysis of the PARK7 SVA's ability to support reporter gene expression and its polymorphic nature. These are properties that would not only be involved in changing the transcriptome of a cell in disease states such as cancer, but also potentially a major driving force in evolution of the hominids.

We have characterised a preferential insertion of SVAs into genic regions (Figure 1), which may reflect the more accessible and open nature of the chromatin to allow for transcription and therefore more amenable to retrotransposon insertions than inactive chromatin. This is reflected in the finding that $62 \%$ of SVAs are within genes or their $10 \mathrm{~kb}$ flank. Waves of SVA retrotransposon integration in the hominids could alter significant number of genes via transcriptional/post transcriptional mechanisms which could act to initiate distinct cascades of gene expression changes which may have major phenotypic affects on cell function. There were also a greater number of SVAs than expected in key regions of the genome such as promoters (Figure 1D), these insertions have placed them where they could potentially influence transcription. The analysis of the prevalence of SVAs upstream of TSS was used to determine that throughout potentially regulatory regions of the genome SVAs are overrepresented. The CG-rich nature of the primary sequence of the SVAs [5] provides potential regions for methylation, many SVAs are located near the transcriptional start site of genes, therefore the methylation status of these elements could influence the expression of the gene as hypothesised for cancer [19,51,52]. Throughout the SVAs, their subtypes and domains share similar primary sequences; which provides the potential for binding similar sequence specific binding factors that could affect aspects of transcription or post transcriptional processing. The end result could be subsets of SVAs which respond to similar cellular signalling pathways which are dependent on chromatin structure.

Primary DNA sequence which contains stretches of tandem guanine nucleotides can fold into four-stranded structures called G4 DNA, which are implicated in gene expression, replication and telomere maintenance [21]. The presence of G4 sequences along with abnormal hypomethylation was shown to be enriched in breakpoints mapped in cancer genomes, leading to the hypothesis that loss of methylation in regions with G4 sequences is part of the mutagenic processes in cancer [25]. Computational analyses using such as the Quadparser programme have suggested these structures are prevalent in the human genome with data demonstrating their function in vitro $[23,26]$. SVAs contain sequences with G4 potential, specifically in their CCCTCT hexamer and central VNTR (Figure 2), therefore could show similar properties to already characterised functions of G4 DNA mentioned previously. Of particular interest would be the hypothesised mutagenic properties of G4 sequences in demethylated regions in cancer as it has been demonstrated that SVAs experience a loss of methylation in cancer [19]. The amount of G4 potential and the domain of the SVA it was predominantly located in varied across the different subtypes. The older subtypes (A, B and C) had the lowest potential; which was mostly located within the 5 ' CCCTCT repeat, whereas the younger human specific (E, F and F1) demonstrated the greatest potential for G4 with an increase in the amount located in the central VNTR. Subtype D showed itself to be an intermediate of the two groups.

The polymorphic nature of SVAs extends to their presence or absence in the genome, this has been analysed for a group of human specific SVAs, it was estimated that $37.5 \%$ of SVA Es and $27.6 \%$ of SVA Fs were polymorphic in their occurrence in the genome [5]. The frequency of this presence or absence of specific SVAs located in $H L A$ genes has shown to be variable between groups with different ethnic origins [53]. This demonstrates the variability of SVA insertions between individuals; our study extends the analysis of their polymorphic nature to include the variation found in the CCCTCT hexamer repeat and provides further evidence of the already characterised variation 
in the second domain of the central VNTR (Table 2). Our data demonstrates the PARK7 SVA has at least four alleles which show variation in the two regions above, which interestingly are also the major regions for potential G4 DNA.

The final parameter we explored was the potential for the SVA to act as a transcriptional regulator in a classical reporter gene model (Figure 4). Although this assay did not allow us to address epigenetic parameters it did allow us to address whether the primary sequence of the SVA could interact with transcription factors to modulate transcriptional properties and further allowed us to delineate potential distinct regulatory domains in the SVA. The definition of the latter was particularly important given the accepted composite nature of domains in SVAs; tandem repeat structures are a class of regulatory DNA which we and others have demonstrated can direct tissue specific and stimulus inducible expression in vitro and in vivo both in mammals and herpes simplex virus $[31,35,54]$. We focused our analysis on the human specific SVA in the promoter of the PARK7 gene. As shown in Figure $4 \mathrm{~B}$ and $\mathrm{C}$ the central TR/VNTR differentially supported reporter gene expression in the two cell lines analysed. It demonstrated repressive qualities in the neuroblastoma cell line SK-N-AS but not in the breast cancer cell line MCF-7 when in the forward orientation. These cell lines were selected they are well characterised and accepted to represent neuronal function (SK-N-AS) and breast cancer (MCF-7) because PARK7, also termed $D J-1$, is associated with both breast cancer and early onset Parkinson's disease [55,56], further they provide preliminary functional data on the ability of the PARK7 SVA to affect expression in different environments. We have previously shown that VNTRs can function in a tissue specific manner so the distinct functions in the cell line models were not unexpected.

The complete SVA showed no activity in the SK-N-AS cell line but enhanced reporter gene expression in MCF7 cells. Interestingly the deletion of the SINE element from the SVA fragment resulted in significantly higher levels of reporter gene expression than the SVA alone in both cell lines. This leads us to postulate that there are probably a minimum of three distinct functional elements in the SVA that adjust its ability to modulate expression, the central TR/VNTR, SINE and the CCCTCT and Alu-like sequences. The data on the central TR/ VNTR indicated they support distinct transcriptional properties dependent on cell type. This is consistent with the action of VNTRs we have previously observed in the human serotonin and dopamine transporter genes $[28,31,34]$. We would expect that different complements of transcription factors present in both these cell lines are responsible for the activity of the reporter gene directed by the TR/VNTR.

\section{Conclusions}

We propose that SVAs have inserted preferentially into genic regions placing them in areas of the genome where they have the potential to affect transcription or post transcriptional regulation through several mechanisms such as methylation state, provision of multiple transcription factor binding sites or formation of DNA secondary structures. We studied the PARK7 SVA in detail, demonstrated its ability to differentially affect transcription within a reporter gene construct in two different cell lines and identified at least four alleles for this particular SVA with multiple regulatory domains. We and others have previously demonstrated the functional consequences, transcriptional properties or utilisation as a biomarker in the human genome for both mental health and cancer of VNTRs. Therefore mechanistically the polymorphic variation we observed can potentially affect several parameters. We also demonstrated in silico that the CCCTCT and central VNTR domains have the potential to form distinct secondary structures (G4), which impart function. There was an increase in the amount of G4 potential, in particularly in the central VNTR, as the SVAs progressed to the younger human specific subtypes as changes occurred in their structure and sequence.

\section{Methods}

\section{Analysis of distribution and structure of SVAs}

A list of SVAs from the repeat masker track of UCSC genome browser (http://genome.ucsc.edu/index.html) with Hg19 was generated and then manually annotated to include any components of the SVA that had not been included. This list along with the UCSC table browser and Galaxy software (https://main.g2.bx.psu.edu/) was used to analyse the distribution of SVAs across the genome. The size and gene content of each chromosome was taken from NCBI human genome overview for 37.3. Quadparser software (http://www.quadruplex.org/) was used to predict the potential of the SVA sequence to form G4 DNA.

\section{Cell culture}

Complete media for SK-N-AS cell line: Dulbecco's Modified Eagles medium with $4500 \mathrm{mg}$ glucose/L (Sigma) supplemented with $1 \%(\mathrm{v} / \mathrm{v})$ non essential amino acid solution (Sigma), 100 units per $\mathrm{ml}$ of penicillin and $0.1 \mathrm{mg} / \mathrm{ml}$ of streptomycin and 10\% (v/v) foetal bovine serum (Sigma). Complete media for MCF-7 cell line: Dulbecco's Modified Eagles medium with $4500 \mathrm{mg}$ glucose/L (Sigma) supplemented with 100 units per $\mathrm{ml}$ of penicillin and $0.1 \mathrm{mg} / \mathrm{ml}$ of streptomycin and $10 \%(\mathrm{v} / \mathrm{v})$ foetal bovine serum (Sigma). Cells were grown in $5 \% \mathrm{CO}_{2}$ and at $37^{\circ} \mathrm{C}$. 


\section{Cloning of PARK7 SVA fragments into PGL3P}

The three fragments of the PARK7 SVA; SVA, SVA wo SINE and the TR/VNTR of the SVA, were amplified using PCR with KOD Hot Start Polymerase (Novagen) under standard conditions with the following primers sets respectively: 5'GGCTTTTTGATAACCCCTGA 3' and 5' TTTCGGATCACAGGCATGAGC 3, 5' GGCTTTTTGAT AACCCCTGA 3' and 5' CCGCCTTTCTATTCCACAAA

3', 5'CTCAGTGCTCAATGGTGCC 3' and 5' CCGC CTTTCTATTCCACAAA 3'. JAr genomic DNA was used as template to amplify the whole SVA and the SVA without the SINE region. The whole SVA amplicon was used in nested PCR to amplify the TR/VNTR of the SVA. These three fragments were sub cloned into an intermediate vector (Zero Blunt PCR vector from Invitrogen) and sequence confirmed by DNA Sequencing and Services, University of Dundee, the fragments corresponded to allele 1. During this cloning process a truncated SVA was generated during one of the transformation steps and this 5 ' fragment was used to produce a fourth reporter gene construct. These intermediate plasmids were firstly digested with restriction enzymes Acc65I and XhoI (Promega) and inserts cloned into the multiple cloning site of pGL3P reporter gene vector upstream of the SV40 minimal promoter (Promega) so that all inserts were in the forward orientation (CCCTCT hexamer at 5' end and poly A-tail at 3' end) and secondly digested with the restriction enzymes BamHI and XbaI, and cloned in to the multiple cloning site of pGL3P which had been digested with Nhe1 and BglII. This resulted in the generation of reporter gene vectors containing the PARK7 SVA fragments in reverse orientation (poly A-tail at 5' end and the CCCTCT hexamer at the 3 ' end).

\section{Transfection of reporter gene constructs and luciferase assay}

The cells were plated out in 24 well plates at the following concentrations $24 \mathrm{hrs}$ prior to transfection: SK-N-AS 120,000 cells per well and MCF-7 100,000 cells per well. Reporter gene constructs $(1 \mu \mathrm{g})$ and internal control TK renilla construct (10 ng) used for normalisation of data, were co transfected using TurboFect (Thermo Scientific) following manufacturers' instructions. Cells were lysed $48 \mathrm{hrs}$ post-transfection and the Dual Lucificerase Reporter Assay (Promega) was performed, luminescence was measured with a Glomax 96 Microplate Luminometer (Promega). Statistical analysis to test the significance of the fold change of the reporter gene constructs over the minimal promoter alone and comparison of forward and reverse orientation fold activity were carried out using a one tailed $t$-test. Significance was scored as follows * $\mathrm{P}<0.05,{ }^{* * *} \mathrm{P}<0.01,{ }^{* * * *} \mathrm{P}<0.001$ and $\# \mathrm{P}<0.05, \# \# \mathrm{P}<0.01$, $\# \# \mathrm{P}<0.001 \mathrm{~N}=4$.

\section{Genotyping PARK7 SVA}

The PARK7 SVA was amplified using the following primer set: forward 5'GGCTTTTTGATAACCCCTGA 3' and reverse 5'GCAAGGCTTAGCTTGGACAG 3' and KOD Hot Start DNA Polymerase (Novagen) under standard conditions with the addition of betaine (Sigma) at $0.5 \mathrm{M}$ final concentration. $1 \mathrm{ng}$ of genomic DNA from the CEU HapMap cohort was used as template. The PCR products were run on $1 \%$ agarose gels stained with GelRed Nucleic Acid Stain (Biotium) and visualised using a UV transilluminator (BioDoc-it Imaging System). Alleles that were difficult to call were repeated and any that remained ambiguous were excluded.

\section{Additional files}

Additional file 1: Gene and SVA density of human chromosomes (.pdf). Data values for graph in Figure 1A showing the SVA and gene densities for each individual chromosome.

Additional file 2: Correlation coefficient of gene and SVA subtype density across human chromosomes (.pdf). A table showing the correlation coefficients between each SVA subtype density and gene density of human chromosomes.

Additional file 3: Distribution of SVA subtypes within $1 \mathrm{~kb}, 10 \mathrm{~kb}$, $20 \mathrm{~kb}$ and $100 \mathrm{~kb}$ upstream of a transcriptional start site (.pdf). A graph comparing the distribution of each SVA subtype in defined regions upstream of transcriptional start sites to their distribution across the whole genome.

\section{Abbreviations}

SVA: SINE-VNTR-Alu; LTRs: Long terminal repeats; LINEs: Long interspersed elements; SINEs: Short interspersed elements; VNTR: Variable number tandem repeat; TR: Tandem repeat; G4: G-quadruplex.

\section{Competing interests}

The authors declare no competing interests.

\section{Authors' contributions}

All authors (ALS, VJB, GB and JPQ) contributed to the design, analysis and interpretation of the study. ALS performed the bioinformatic analysis, reporter gene assays and genotyping. ALS and GB completed statistical analysis. All authors contributed to the production of the manuscript. All authors read and approved the final manuscript.

\section{Acknowledgments}

This work was funded by the University of Liverpool Demonstratorship program (ALS). The authors acknowledge Veridiana Miyajima and lain Kirk for technical assistance and Alix Warburton, Kate Haddley and Paul Myers for their support and advice.

Lab work funded in part by the National Institute for Health Research (NIHR) Biomedical Research Centre for Mental Health at South London and Maudsley NHS Foundation Trust and [Institute of Psychiatry] King's College London. This article/paper/report presents independent research in part funded by the National Institute for Health Research (NIHR). The views expressed are those of the author(s) and not necessarily those of the NHS, the NIHR or the Department of Health.

\section{Author details}

${ }^{1}$ Department of Molecular and Clinical Pharmacology, Institute of Translational Medicine, The University of Liverpool, Liverpool L69 3BX, UK. ${ }^{2}$ King's College London, MRC Social Genetic and Developmental Psychiatry Research Centre, Institute of Psychiatry, London, UK. ${ }^{3}$ National Institute for Health Research (NIHR) Biomedical Research Centre for Mental Health, South London and Maudsley NHS Foundation Trust and Institute of Psychiatry, King's College London SE5 8DF, UK. 
Received: 25 February 2013 Accepted: 15 May 2013

Published: 21 May 2013

\section{References}

1. Ono M, Kawakami M, Takezawa T: A novel human nonviral retroposon derived from an endogenous retrovirus. Nucleic Acids Res 1987, 15:8725-8737.

2. Zhu ZB, Hsieh SL, Bentley DR, Campbell RD, Volanakis JE: A variable number of tandem repeats locus within the human complement C2 gene is associated with a retroposon derived from a human endogenous retrovirus. J Exp Med 1992, 175:1783-1787.

3. Shen L, Wu LC, Sanlioglu S, Chen R, Mendoza AR, Dangel AW, Carroll MC, Zipf WB, Yu CY: Structure and genetics of the partially duplicated gene $\mathrm{RP}$ located immediately upstream of the complement $\mathrm{C} 4 \mathrm{~A}$ and the $\mathrm{C} 4 \mathrm{~B}$ genes in the HLA class III region. Molecular cloning, exon-intron structure, composite retroposon, and breakpoint of gene duplication. J Biol Chem 1994, 269:8466-8476.

4. Han K, Konkel MK, Xing J, Wang H, Lee J, Meyer TJ, Huang CT, Sandifer E, Hebert K, Barnes EW, et al: Mobile DNA in Old world monkeys: a glimpse through the rhesus macaque genome. Science 2007, 316:238-240.

5. Wang H, Xing J, Grover D, Hedges DJ, Han K, Walker JA, Batzer MA: SVA elements: a hominid-specific retroposon family. J Mol Biol 2005, 354:994-1007.

6. Bantysh $O B$, Buzdin AA: Novel family of human transposable elements formed due to fusion of the first exon of gene MAST2 with retrotransposon SVA. Biochemistry (MosC) 2009, 74:1393-1399.

7. Hancks DC, Ewing AD, Chen JE, Tokunaga K, Kazazian HH Jr: Exon-trapping mediated by the human retrotransposon SVA. Genome Res 2009, 19:1983-1991.

8. Damert A, Raiz J, Horn AV, Lower J, Wang H, Xing J, Batzer MA, Lower R, Schumann GG: 5'-Transducing SVA retrotransposon groups spread efficiently throughout the human genome. Genome Res 2009, 19:1992-2008.

9. Zabolotneva AA, Bantysh O, Suntsova MV, Efimova N, Malakhova GV, Schumann GG, Gayfullin NM, Buzdin AA: Transcriptional regulation of human-specific SVAF(1) retrotransposons by cis-regulatory MAST2 sequences. Gene 2012, 505:128-136.

10. Hancks DC, Goodier JL, Mandal PK, Cheung LE, Kazazian HH Jr: Retrotransposition of marked SVA elements by human L1s in cultured cells. Hum Mol Genet 2011, 20:3386-3400.

11. Raiz J, Damert A, Chira S, Held U, Klawitter S, Hamdorf M, Lower J, Stratling WH, Lower R, Schumann GG: The non-autonomous retrotransposon SVA is trans-mobilized by the human LINE-1 protein machinery. Nucleic Acids Res 2012, 40:1666-1683.

12. Xing J, Zhang Y, Han K, Salem AH, Sen SK, Huff CD, Zhou Q, Kirkness EF, Levy S, Batzer MA, Jorde LB: Mobile elements create structural variation: analysis of a complete human genome. Genome Res 2009, 19:1516-1526.

13. Hancks DC, Mandal PK, Cheung LE, Kazazian HH Jr: The minimal active human SVA retrotransposon requires only the 5'-hexamer and Alu-like domains. Mol Cell Biol 2012, 32:4718-4726.

14. Hancks DC, Kazazian HH Jr: Active human retrotransposons: variation and disease. Curr Opin Genet Dev 2012, 22:191-203.

15. van der Klift HM, Tops CM, Hes FJ, Devilee P, Wijnen JT: Insertion of an SVA element, a nonautonomous retrotransposon, in PMS2 intron 7 as a novel cause of Lynch syndrome. Hum Mutat 2012, 33:1051-1055.

16. Watanabe M, Kobayashi K, Jin F, Park KS, Yamada T, Tokunaga K, Toda T: Founder SVA retrotransposal insertion in Fukuyama-type congenital muscular dystrophy and its origin in Japanese and Northeast Asian populations. Am J Med Genet A 2005, 138:344-348.

17. Takasu M, Hayashi R, Maruya E, Ota M, Imura K, Kougo K, Kobayashi C, Saji H, Ishikawa Y, Asai T, Tokunaga K: Deletion of entire HLA-A gene accompanied by an insertion of a retrotransposon. Tissue Antigens 2007, 70:144-150

18. Baillie JK, Barnett MW, Upton KR, Gerhardt DJ, Richmond TA, De Sapio F, Brennan PM, Rizzu P, Smith S, Fell M, et al: Somatic retrotransposition alters the genetic landscape of the human brain. Nature 2011, 479:534-537.

19. Szpakowski S, Sun X, Lage JM, Dyer A, Rubinstein J, Kowalski D, Sasaki C, Costa J, Lizardi PM: Loss of epigenetic silencing in tumors preferentially affects primate-specific retroelements. Gene 2009, 448:151-167.

20. Hancks DC, Kazazian HH Jr: SVA retrotransposons: Evolution and genetic instability. Semin Cancer Biol 2010, 20:234-245.

21. Nakagama H, Higuchi K, Tanaka E, Tsuchiya N, Nakashima K, Katahira M, Fukuda H: Molecular mechanisms for maintenance of G-rich short tandem repeats capable of adopting G4 DNA structures. Mutat Res 2006, 598:120-131.

22. de Messieres M, Chang JC, Brawn-Cinani B, La Porta A: Single-molecule study of g-quadruplex disruption using dynamic force spectroscopy. Phys Rev Lett 2012, 109:058101.

23. Membrino A, Cogoi S, Pedersen EB, Xodo LE: G4-DNA formation in the HRAS promoter and rational design of decoy oligonucleotides for cancer therapy. PLoS One 2011, 6:e24421.

24. Clark DW, Phang T, Edwards MG, Geraci MW, Gillespie MN: Promoter G-quadruplex sequences are targets for base oxidation and strand cleavage during hypoxia-induced transcription. Free Radic Biol Med 2012, 53:51-59.

25. De S, Michor F: DNA secondary structures and epigenetic determinants of cancer genome evolution. Nat Struct Mol Biol 2011, 18:950-955.

26. Huppert $\lrcorner$, Balasubramanian S: Prevalence of quadruplexes in the human genome. Nucleic Acids Res 2005, 33:2908-2916.

27. Fletcher TM, Sun D, Salazar M, Hurley LH: Effect of DNA secondary structure on human telomerase activity. Biochemistry 1998, 37:5536-5541.

28. Vasiliou SA, Ali FR, Haddley K, Cardoso MC, Bubb VJ, Quinn JP: The SLC6A4 VNTR genotype determines transcription factor binding and epigenetic variation of this gene in response to cocaine in vitro. Addict Biol 2012, 17:156-170

29. Haddley K, Bubb VJ, Breen G, Parades-Esquivel UM, Quinn JP: Behavioural Genetics of the Serotonin Transporter. Curr Top Behav Neurosci 2012.

30. Brotons O, O'Daly OG, Guindalini C, Howard M, Bubb J, Barker G, Dalton J, Quinn J, Murray RM, Breen G, Shergill SS: Modulation of orbitofrontal response to amphetamine by a functional variant of DAT1 and in vitro confirmation. Mol Psychiatry 2011, 16:124-126.

31. Ali FR, Vasiliou SA, Haddley K, Paredes UM, Roberts JC, Miyajima F, Klenova E, Bubb VJ, Quinn JP: Combinatorial interaction between two human serotonin transporter gene variable number tandem repeats and their regulation by CTCF. J Neurochem 2010, 112:296-306.

32. Breen G, Collier D, Craig I, Quinn J: Variable number tandem repeats as agents of functional regulation in the genome. IEEE Eng Med Biol Mag 2008, 27:103-104. 108

33. Roberts J, Scott AC, Howard MR, Breen G, Bubb VJ, Klenova E, Quinn JP: Differential regulation of the serotonin transporter gene by lithium is mediated by transcription factors, CCCTC binding protein and Y-box binding protein 1, through the polymorphic intron 2 variable number tandem repeat. J Neurosci 2007, 27:2793-2801.

34. Guindalini C, Howard M, Haddley K, Laranjeira R, Collier D, Ammar N, Craig I, O'Gara C, Bubb VJ, Greenwood T, et al: A dopamine transporter gene functional variant associated with cocaine abuse in a Brazilian sample. Proc Natl Acad Sci USA 2006, 103:4552-4557.

35. Mackenzie A, Quinn J: A serotonin transporter gene intron 2 polymorphic region, correlated with affective disorders, has allele-dependent differential enhancer- like properties in the mouse embryo. Proc Natl Acad Sci USA 1999, 96:15251-15255.

36. Schwarzenbach H, Goekkurt E, Pantel K, Aust DE, Stoehlmacher J: Molecular analysis of the polymorphisms of thymidylate synthase on cell-free circulating DNA in blood of patients with advanced colorectal carcinoma. Int J Cancer 2010, 127:881-888.

37. Lee SY, Hahn CY, Lee JF, Chen SL, Chen SH, Yeh TL, Kuo PH, Lee IH, Yang YK, Huang SY, et al: MAOA-uVNTR polymorphism may modify the protective effect of ALDH2 gene against alcohol dependence in antisocial personality disorder. Alcohol Clin Exp Res 2009, 33:985-990.

38. Munafo MR, Johnstone EC: Smoking status moderates the association of the dopamine D4 receptor (DRD4) gene VNTR polymorphism with selective processing of smoking-related cues. Addict Biol 2008, 13:435-439.

39. Herman Al, Kaiss KM, Ma R, Philbeck JW, Hasan A, Dasti H, DePetrillo PB: Serotonin transporter promoter polymorphism and monoamine oxidase type A VNTR allelic variants together influence alcohol binge drinking risk in young women. Am J Med Genet B Neuropsychiatr Genet 2005, 133:74-78.

40. Lowe N, Kirley A, Mullins C, Fitzgerald M, Gill M, Hawi Z: Multiple marker analysis at the promoter region of the DRD4 gene and ADHD: evidence of linkage and association with the SNP -616. Am J Med Genet B Neuropsychiatr Genet 2004, 131:33-37.

41. Coutinho AM, Oliveira G, Morgadinho T, Fesel C, Macedo TR, Bento C, Marques C, Ataide A, Miguel T, Borges L, Vicente AM: Variants of the 
serotonin transporter gene (SLC6A4) significantly contribute to hyperserotonemia in autism. Mol Psychiatry 2004, 9:264-271.

42. Hranilovic D, Stefulj J, Furac I, Kubat M, Balija M, Jernej B: Serotonin transporter gene promoter (5-HTTLPR) and intron 2 (VNTR) polymorphisms in Croatian suicide victims. Biol Psychiatry 2003, 54:884-889.

43. Anguelova M, Benkelfat C, Turecki G: A systematic review of association studies investigating genes coding for serotonin receptors and the serotonin transporter: I. Affective disorders. Mol Psychiatry 2003, 8:574-591.

44. Visel A, Rubin EM, Pennacchio LA: Genomic views of distant-acting enhancers. Nature 2009, 461:199-205.

45. Shanley L, Davidson S, Lear M, Thotakura AK, McEwan IJ, Ross RA, MacKenzie A: Long-range regulatory synergy is required to allow control of the TAC1 locus by MEK/ERK signalling in sensory neurones. Neurosignals 2010, 18:173-185.

46. Wong HM, Stegle O, Rodgers S, Huppert JL: A toolbox for predicting gquadruplex formation and stability. J Nucleic Acids 2010, 2010.

47. Taira T, Takahashi K, Kitagawa R, Iguchi-Ariga SM, Ariga H: Molecular cloning of human and mouse DJ-1 genes and identification of Sp1-dependent activation of the human DJ-1 promoter. Gene 2001, 263:285-292.

48. Kazazian HH Jr, Wong C, Youssoufian H, Scott AF, Phillips DG, Antonarakis SE: Haemophilia $A$ resulting from de novo insertion of $L 1$ sequences represents a novel mechanism for mutation in man. Nature 1988, 332:164-166.

49. Wilund KR, Yi M, Campagna F, Arca M, Zuliani G, Fellin R, Ho YK, Garcia JV, Hobbs HH, Cohen JC: Molecular mechanisms of autosomal recessive hypercholesterolemia. Hum Mol Genet 2002, 11:3019-3030.

50. Haddley K, Vasiliou AS, Ali FR, Paredes UM, Bubb VJ, Quinn JP: Molecular genetics of monoamine transporters: relevance to brain disorders. Neurochem Res 2008, 33:652-667.

51. Park JH, Park J, Choi JK, Lyu J, Bae MG, Lee YG, Bae JB, Park DY, Yang HK, Kim TY, Kim YJ: Identification of DNA methylation changes associated with human gastric cancer. BMC Med Genomics 2011, 4:82.

52. Konkel MK, Batzer MA: A mobile threat to genome stability: The impact of non-LTR retrotransposons upon the human genome. Semin Cancer Biol 2010, 20:211-221.

53. Kulski JK, Shigenari A, Inoko H: Polymorphic SVA retrotransposons at four loci and their association with classical HLA class I alleles in Japanese, Caucasians and African Americans. Immunogenetics 2010, 62:211-230.

54. Stevens HC, Fiskerstrand C, Bubb VJ, Dalziel R, Quinn JP: A regulatory domain spanning the repeat sequence RE1 from herpes simplex virus type 1 has cell specific differential functions in trigeminal neurons and fibroblasts. FEBS Lett 2009, 583:3335-3338.

55. Bonifati V, Rizzu P, Squitieri F, Krieger E, Vanacore N, van Swieten JC, Brice A, van Duijn CM, Oostra B, Meco G, Heutink P: DJ-1(PARK7), a novel gene for autosomal recessive, early onset parkinsonism. Neurol Sci 2003, 24:159-160.

56. Le Naour F, Misek DE, Krause MC, Deneux L, Giordano TJ, Scholl S, Hanash SM: Proteomics-based identification of RS/DJ-1 as a novel circulating tumor antigen in breast cancer. Clin Cancer Res 2001, 7:3328-3335.

doi:10.1186/1471-2148-13-101

Cite this article as: Savage et al:: Characterisation of the potential function of SVA retrotransposons to modulate gene expression patterns. BMC Evolutionary Biology 2013 13:101.

\section{Submit your next manuscript to BioMed Central and take full advantage of:}

- Convenient online submission

- Thorough peer review

- No space constraints or color figure charges

- Immediate publication on acceptance

- Inclusion in PubMed, CAS, Scopus and Google Scholar

- Research which is freely available for redistribution 